\title{
Limits for zeros of Jacobi and Laguerre polynomias
}

\author{
Fábio R. Lucas* \\ Depto de Matemática, Campus de Nova Andradina, UEMS, \\ 79750-000, Nova Andradina, MS \\ E-mail: fabiorodrigues@uems.br
}

\begin{abstract}
$\underline{\text { Abstract }}$
Denote by $P_{n}^{(\alpha, \beta)}(x)$ and $L_{n}^{\alpha}(x)$ the classical Jacobi and Laguerre polynomials. In a recent paper Driver and Jordaan developed a method to obtain limits for zeros of orthogonal polynomials and applied it for the zeros of Jacobi and Laguerre polynomials. We show how to refine the method to obtain sharper limits for the same zeros. It turns out that the new limits obtained in this note are very precise.
\end{abstract}

\section{Introduction}

Let $\left\{p_{n}\right\}_{n=0}^{\infty}$ be any sequence of orthogonal polynomials. Then it is well known that the zeros of $p_{n}$ are real, simple and interlace with the zeros of $p_{n-1}$. Denote by $w_{n}<\ldots<w_{1}$ the zeros of Jacobi Polynomials $P_{n}^{(\alpha, \beta)}(x)$ and $y_{n}<\ldots<y_{1}$ the zeros of Laguerre Polynomials $L_{n}^{\alpha}(x)$.

Driver and Jordaan [5] established the following interesting result:

Theorem A. Let $\left\{p_{n}\right\}_{n=0}^{\infty}$ be a sequence of polynomials, orthogonal in $(c, d)$ with respect to a positive Borel measure.

Let $g_{n-k}$ be a polynomial of degree $n-k-1$ which satisfies, for any $k<n$ and $n \in \mathbb{N}$,

$$
f(x) g_{n-k}(x)=G_{k}(x) p_{n-1}(x)+H(x) p_{n}(x)
$$

where $f(x) \neq 0$ for $x \in(c, d)$, and $H(x)$ and $G_{k}(x)$ are polynomials with $\operatorname{deg}\left(G_{k}\right)=k$. Then, for any fixed $k \in\{1, \ldots, n-1\}$ and $n \in \mathbb{N}$, the $n-1$ real and simple zeros of $G_{k} g_{n-k}$ interlace with the zeros of $p_{n}$ if $g_{n-k}$ and $p_{n}$ are co-primes.

Corollary A. Suppose (1) holds for $k, n \in \mathbb{N}$ fixed and $k<n-1$. The largest (smallest) zero of $G_{k}$ is a strict lower (upper) bound for the largest (smallest) zero of $p_{n}$.

In (1) we set $p_{n}=P_{n}^{(\alpha, \beta)}(x)$ and $p_{n-1}=P_{n-1}^{(\alpha, \beta)}(x)$ and obtain

$$
f(x) g_{n-k}(x)=G_{k}(x) P_{n-1}^{(\alpha, \beta)}(x)+H(x) P_{n}^{(\alpha, \beta)}(x) .
$$

Therefore, by Corollary A, we need to find the largest and the smallest zeros of $G_{k}$ in order to be able to limit the extreme zeros of the Jacobi polynomial from "inside" that is, to obtain lower limit for the largest zero $w_{1}$ and upper limit for the smallest zero $w_{n}$ of $P_{n}^{(\alpha, \beta)}(x)$.

For Jacobi polynomials $P_{n}^{(\alpha, \beta)}, \alpha, \beta>-1$, it was proved in [3], Theorem 2.1(i)(c)] that (1) holds for $k=1$ with

$$
\begin{gathered}
G_{n-1}=P_{n-2}^{\alpha+4, \beta}, \quad G_{1}(x)=x-A_{n}, \\
A_{n}=\frac{2(n-1)(n+\alpha+\beta+2)+(\alpha+3)(\beta-\alpha)}{2(n-1)(n+\alpha+\beta+2)+(\alpha+3)(\alpha+\beta+2)} \text { and } p_{n}=P_{n}^{(\alpha, \beta)},
\end{gathered}
$$

${ }^{*}$ FAPESP 
for $n>1, n \in \mathbb{N}$. It follows from Corollary $\mathrm{A}$, that for all $\alpha, \beta>-1, n \in \mathbb{N}$,

$$
w_{1}>1-\frac{2(\alpha+1)(\alpha+3)}{2(n-1)(n+\alpha+\beta+2)+(\alpha+3)(\alpha+\beta+2)}=1-\mathcal{O}\left(\frac{1}{n^{2}}\right)
$$

which is better than $w_{1}>1-2(\alpha+1) /(2 n+\alpha+\beta)$, obtained by Szegô in [6].

Since $P_{n}^{(\alpha, \beta)}(x)=(-1)^{n} P_{n}^{(\beta, \alpha)}(-x)$, then (3) yields

$$
w_{n}<-1+\frac{2(\beta+1)(\beta+3)}{2(n-1)(n+\alpha+\beta+2)+(\beta+3)(\alpha+\beta+2)}=1-\mathcal{O}\left(\frac{1}{n^{2}}\right) .
$$

For $\alpha>-1$, the Laguerre polynomials $L_{n}^{\alpha}$ satisty the mixed three term recurrence relation

$$
\begin{aligned}
x^{5} L_{n-3}^{\alpha+5}(x)= & (n+\alpha)(\alpha+1)_{4}-(\alpha+2)_{2}(3 n+2 \alpha+2) x \\
& +(n+\alpha+1)_{2} x^{2} L_{n-1}^{\alpha}(x)+H(x) L_{n}^{\alpha}(x)
\end{aligned}
$$

which follows from [4], Eq. (13) and the three term recurrence relation for Laguerre polynomials (cf.[6]), here, $(\alpha)_{k}=\alpha(\alpha+1) \ldots(\alpha+k-1), k \in \mathbb{N}$, is Pochhammer symbol.

For the largest zero $y_{1}$ and smallest zero $y_{n}$ of the classical Laguerre polynomial $L_{n}^{\alpha}(x)$, Driver and Jordaan obtained the following limits using Corollary A and equation (5)

$$
y_{1}>2 n+\alpha-2+\sqrt{n^{2}+n(\alpha-2)-(\alpha-2)}
$$

which is again better than the limit $2 n+\alpha-1$ found in Szegó's book [6], but when $n \rightarrow \infty$ it is worse than the bound $4 n+\alpha-16 \sqrt{2 n}$, proved by Bottema [1]. Driver and Jordan proved that

$$
y_{n}<\frac{(\alpha+2)_{2}(3 n+2 \alpha+2)-B_{n}}{2(n+\alpha+1)_{2}}
$$

where

$$
B_{n}=\sqrt{(\alpha+2)_{2}\left(-4(\alpha+1)^{2}(\alpha+2)+T\right)}
$$

and

$$
T=4 n(\alpha+1)\left(\alpha^{2}+4 \alpha+6\right)+\left(5 \alpha^{2}+25 \alpha+38\right) n^{2} .
$$

In the next section we give the method used to proving the following theorem:

Theorem 1. Denote by $w_{n}<\ldots<w_{1}$ the zeros of the Jacobi polynomial $P_{n}^{(\alpha, \beta)}(x)$ and $y_{n}<\ldots<y_{1}$ the zeros of the Laguerre polynomial $L_{n}^{\alpha}(x)$. Then

$$
w_{n}<-1+\frac{2(\beta+1)(\beta+2)(\beta+3)}{\widetilde{D}+\widetilde{E}}, \quad 1-\frac{2(\alpha+1)(\alpha+2)(\alpha+3)}{D+E}<w_{1}
$$

and

$$
\begin{gathered}
y_{n}<\frac{(\alpha+1)(\alpha+2)(\alpha+3)}{(\alpha+2)(\alpha+n+1)+\sqrt{(\alpha+2)(\alpha+n+1)(-1-\alpha+n(2+\alpha))}}, \\
2 n+\alpha-2+\sqrt{n^{2}+n(\alpha-2)-(\alpha-2)}<y_{1}
\end{gathered}
$$

where

$$
D=n^{2}(\alpha+2)+(\alpha+1)(\alpha+2)(\alpha+\beta+1)+n(2+\alpha)(2+2 \alpha+\beta)
$$

and

$E=\sqrt{(\alpha+2)(\alpha+n+1)(\alpha+\beta+n+1)\left(-2 n(\alpha+1)+n^{2}(\alpha+2)-(\alpha+1)(\beta-2)+n(\alpha+2) \beta\right)}$,

and $\widetilde{D}$ and $\widetilde{E}$ are obtained from $D$ and $E$ interchanging the roles of $\alpha$ and $\beta$. 


\section{Polynomials generated by the Euclidean Algorithm.}

Consider the polynomials with real coefficients

$$
\begin{aligned}
& f(z)=a_{n} z^{n}+a_{n-1} z^{n-1}+\ldots+a_{1} z+a_{0}, a_{n}=1, \\
& g(z)=b_{n-1} z^{n-1}+\ldots+b_{1} z+b_{0} .
\end{aligned}
$$

With the pair of polynomials $f$ and $g$ we associate the so-called Hurwitz matrix of order $2 n-1$,

$$
\mathcal{H}_{2 n-1}(g, f)=\left(\begin{array}{cccccccc}
b_{n-1} & b_{n-2} & \ldots & b_{0} & 0 & \ldots & 0 & 0 \\
a_{n} & a_{n-1} & \ldots & a_{1} & a_{0} & \ldots & 0 & 0 \\
0 & b_{n-1} & \ldots & b_{1} & b_{0} & \ldots & 0 & 0 \\
0 & a_{n} & \ldots & a_{2} & a_{1} & \ldots & 0 & 0 \\
\vdots & \vdots & & \vdots & \vdots & & \vdots & \vdots \\
0 & 0 & \ldots & a_{n-1} & a_{n-2} & \ldots & a_{1} & a_{0} \\
0 & 0 & \ldots & b_{n-1} & b_{n-2} & \ldots & b_{1} & b_{0}
\end{array}\right)
$$

Denote by

$$
\mathcal{H}_{2 r-1}(g, f)\left(\begin{array}{cccc}
1 & \ldots & 2 r-2 & 2 r-1 \\
1 & \ldots & 2 r-2 & 2 r-1+l
\end{array}\right)
$$

the principal matrix of $\mathcal{H}_{2 n-1}(g, f)$ of order $2 r-1$, formed by the first $2 r-1$ rows and the first $2 r-2$ columns, together with the column $2 r-1+l$. Then

$$
\nabla_{2 r-1}^{(l)}=\nabla_{2 r-1}^{(l)}\left(\begin{array}{cccc}
1 & \ldots & 2 r-2 & 2 r-1 \\
1 & \ldots & 2 r-2 & 2 r-1+l
\end{array}\right)
$$

denotes the determinant

$$
\operatorname{det}\left(\mathcal{H}_{2 r-1}(g, f)\left(\begin{array}{cccc}
1 & \ldots & 2 r-2 & 2 r-1 \\
1 & \ldots & 2 r-2 & 2 r-1+l
\end{array}\right)\right) .
$$

In particularly, by $\nabla_{2 r-1}$ we mean the determinant $\nabla_{2 r-1}^{(0)}$, which is the principal minor of order $2 r-1$ of $\mathcal{H}_{2 n-1}(g, f)$.

Define $Q_{n}(x)=f(x)$ and $Q_{n-1}(x)=g(x)$. Then the algorithm of Euclides generates the polynomials $Q_{n-2}(x), Q_{n-3}(x), \ldots, Q_{0}(x)$ as follows

$$
Q_{n+1-r}(x)=\left(\alpha_{r} x+\beta_{r}\right) Q_{n-r}(x)-Q_{n-1-r}(x), \quad r=1,2, \ldots, n-1 .
$$

In [2], D.K. Dimitrov, F.R. Lucas and A. S. Ranga obtained all polynomials generated by the Euclidean Algorithm as follows:

Theorem B. Lets $f(z)$ and $g(z)$ be defined as above. Then the polynomials $Q_{n-r}(z), r=2,3, \ldots n$, generated by the Euclidean algorithm are given by

$$
Q_{n-r}(z)=M_{r}(z) f(z)+N_{r}(z) g(z),
$$

where $M_{r}$ is a polynomial of degree $r-2, N_{r}$ is a polynomial of degree $r-1$ and

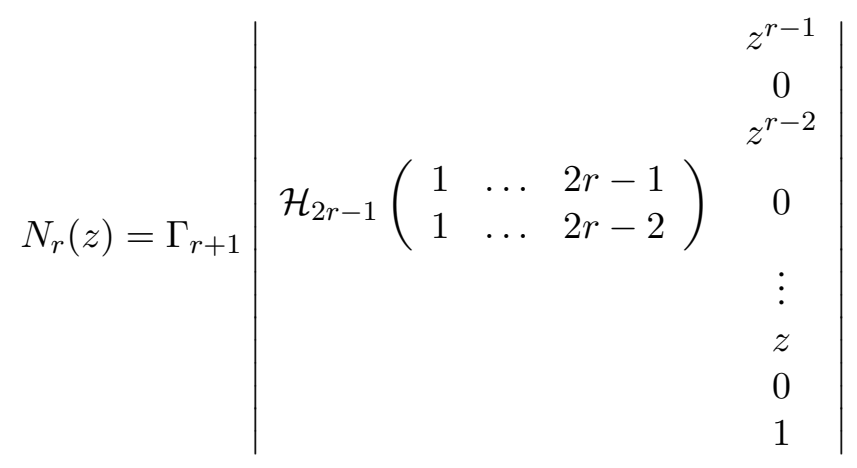


and

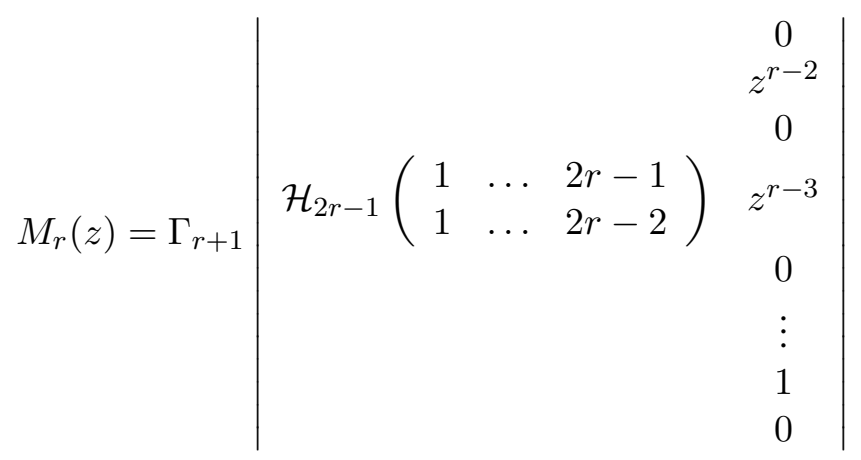

where

$$
\Gamma_{r+1}=\left[\nabla_{2 r-3}\right]^{-2}\left[\nabla_{2 r-5}\right]^{2}\left[\nabla_{2 r-7}\right]^{-2}\left[\nabla_{2 r-9}\right]^{2} \ldots\left[\nabla_{3}\right]^{2(-1)^{r}}\left[\nabla_{1}\right]^{2(-1)^{r+1}} .
$$

In order to combine the results in Theorems $\mathrm{A}$ and $\mathrm{B}$, first we set $r=k+1$ in (9) and consider $f(x)=$ $p_{n}(x)$ and $g(x)=p_{n-1}(x)$, which are two polynomials with interlacing zeros. Thus

$$
Q_{n-k-1}(x)=M_{k+1}(x) p_{n}(x)+N_{k+1}(x) p_{n-1}(z), \quad k=1,2, \ldots n
$$

where the degree of $M_{k+1}$ is $k-1$ and the degree of $N_{k+1}$ is $k$.

Then Theorem A becomes equivalent to Theorem B, with $f(x)=1$ and $G_{k}(x)=N_{k+1}(x)$.

In order to calculate the largest and the smallest zeros of $G_{k}(x)=N_{k+1}(x)$, which is explicitly given by the formula (10), we shall calculate the zeros of the polynomial $N_{k+1}(x)$.

\section{Jacobi Polynomials}

In (12), setting $k=2, p_{n}=P_{n}^{(\alpha, \beta)}$ and $p_{n-1}=P_{n-1}^{(\alpha, \beta)}$ and using the explicit representations

$$
\frac{n !}{(\alpha+1)_{n}} P_{n}^{(\alpha, \beta)}(1-2 x)=F_{2,1}(-n, 1+\alpha+\beta+n ; \alpha+1 ; x)=\sum_{k=0}^{n} a_{k} x^{k}
$$

and

$$
\frac{(n-1) !}{(\alpha+1)_{n-1}} P_{n-1}^{(\alpha, \beta)}(1-2 x)=F_{2,1}(-(n-1), \alpha+\beta+n ; \alpha+1 ; x)=\sum_{k=0}^{n-1} b_{k} x^{k},
$$

by (10), we obtain

$$
N_{3}(x)=\left|\begin{array}{ccccc}
b_{n-1} & b_{n-2} & b_{n-3} & b_{n-4} & x^{2} \\
a_{n} & a_{n-1} & a_{n-2} & a_{n-3} & 0 \\
0 & b_{n-1} & b_{n-2} & b_{n-3} & x \\
0 & a_{n} & a_{n-1} & a_{n-2} & 0 \\
0 & 0 & b_{n-1} & b_{n-1} & 1
\end{array}\right|
$$

Hence $N_{3}(x)=A x^{2}+B x+C$, where the coefficients $A, B, C$ are given by

$$
\begin{aligned}
A= & 6+11 \alpha+6 \alpha^{2}+\alpha^{3}, \\
B= & 4+8 n+4 n^{2}+10 \alpha+12 n \alpha+2 n^{2} \alpha+8 \alpha^{2}+4 n \alpha^{2}+2 \alpha^{3}+4 \beta+n \beta \\
& +6 \alpha \beta+2 n \alpha \beta+2 \alpha^{2} \beta, \\
C= & 2 n+4 n^{2}+2 n^{3}+\alpha+6 n \alpha+5 n^{2} \alpha+2 \alpha^{2}+4 n \alpha^{2}+\alpha^{3}+\beta+4 n \beta \\
& +3 n^{2} \beta+3 \alpha \beta+5 n \alpha \beta+2 \alpha^{2} \beta+\beta^{2}+n \beta^{2}+\alpha \beta^{2} .
\end{aligned}
$$


Since all the calculation are done with $P_{n}^{(\alpha, \beta)}(1-2 x)$ and we are interested in the extreme zeros of $N_{3}(x)$ related to the Jacobi polynomial $P_{n}^{(\alpha, \beta)}(x)$. For calculating the zeros $x_{1}$ and $x_{2}$ of $N_{3}(x)$ we have to perform a change of variables $z_{1}=2 / x_{1}+1$ and $z_{2}=2 / x_{2}+1$. Straightforward calculations yield

$$
z_{1}=1-\frac{2(\alpha+1)(\alpha+2)(\alpha+3)}{D-E}
$$

and

$$
z_{2}=1-\frac{2(\alpha+1)(\alpha+2)(\alpha+3)}{D+E}
$$

where

$$
D=n^{2}(\alpha+2)+(\alpha+1)(\alpha+2)(\alpha+\beta+1)+n(2+\alpha)(2+2 \alpha+\beta)
$$

and

$$
E=\sqrt{(\alpha+2)(\alpha+n+1)(\alpha+\beta+n+1)\left(-2 n(\alpha+1)+n^{2}(\alpha+2)-(\alpha+1)(\beta-2)+n(\alpha+2) \beta\right)} .
$$

Therefore by Corollary A, $z_{2}$ is lower limit for the largest zero $w_{1}$ of Jacobi Polynomials.

It is not difficult to see that the limit $z_{2}$ is better than the one provided by Driver and Jordaan in (3). To check this assertion it suffices to show that the difference

$$
\frac{1}{2(n-1)(n+\alpha+\beta+2)+(\alpha+3)(\alpha+\beta+2)}-\frac{1}{\frac{D+E}{\alpha+2}}
$$

is always positive. Thus (14) is equivalent to show that the denominator of the second fraction is greater than the denominator of the first fraction. In other words

$$
\frac{D+E}{\alpha+2}>2(n-1)(n+\alpha+\beta+2)+(\alpha+3)(\alpha+\beta+2) .
$$

Writing (15) as an inequality for E, we obtain after some simplifications

$$
(\alpha+1)^{2}(n-1)(n+\beta-1)(\alpha+\beta+2 n)>0,
$$

which is true for all $\alpha, \beta>-1$ and $n \geq 2$ thus proving our claim. We will omit the proof of the other cases because the proof is analogous to this one.

Since the zeros of the Jacobi polynomials are symmetric when changing the parameters $\alpha$ and $\beta$, if we set

$$
\widetilde{z_{2}}=-1+\frac{2(\beta+1)(\beta+2)(\beta+3)}{\widetilde{D}+\widetilde{E}},
$$

where

$$
\widetilde{D}=n^{2}(\beta+2)+(\beta+1)(\beta+2)(\alpha+\beta+1)+n(2+\beta)(2+2 \beta+\alpha)
$$

and

$\widetilde{E}=\sqrt{(\beta+2)(\beta+n+1)(\alpha+\beta+n+1)\left(-2 n(\beta+1)+n^{2}(\beta+2)-(\beta+1)(\alpha-2)+n(\beta+2) \alpha\right)}$

then $w_{n}<-\widetilde{z_{2}}$ which equivalent to Theorem 1. The latter is better than the one given in (4).

\section{Laguerre Polynomials}

lets $w_{n}$ and $w_{1}$ be the smallest and the largest zeros, respectively, of the Jacobi polynomial, and that $y_{n}$ and $y_{1}$ be the smallest and the largest zeros of the Laguerre polynomial, it is known that

$$
\lim _{\beta \rightarrow \infty} \frac{\beta}{2}\left(1-w_{n}\right)=y_{1}
$$


and

$$
\lim _{\beta \rightarrow \infty} \frac{\beta}{2}\left(1-w_{1}\right)=y_{n}
$$

If we perform the limit $\lim _{\beta \rightarrow \infty} \frac{\beta}{2}\left(1-\widetilde{z_{2}}\right)$ in (17) where $\widetilde{z_{2}}$ is given in (16) we obtain the limit

$$
2 n-2+\alpha+\sqrt{2+n^{2}+n(\alpha-2)-\alpha}<y_{1}
$$

which is the same lower limit, obtained by Driver and Jordaan in (6).

Performing the limit $\lim _{\beta \rightarrow \infty} \frac{\beta}{2}\left(1-z_{2}\right)$ in (18) where $z_{2}$ is given in (13) we obtain the limit

$$
y_{n}<\frac{(\alpha+1)(\alpha+2)(\alpha+3)}{(\alpha+2)(\alpha+n+1)+\sqrt{(\alpha+2)(\alpha+n+1)(-1-\alpha+n(2+\alpha))}}
$$

which is better that the lower limit, obtained by Driver and Jordaan in (7).

keywords: Zeros of orthogonal polynomials, Jacobi polynomials, Laguerre polynomials

\section{References}

[1] O. Bоттемa, Die Nullstellen gewisser durch Rekursionsformeln definierter Polynome, Proc. Amsterdam 34 (5) (1931) 681-691.

[2] D.K. Dimitrov, F.R. Lucas and A. S. Ranga, Sturm Polynomials and Hurwitz Matrices, (submitted).

[3] K.Driver, A. Jooste, K. JordaAn, Stieltjes interlacing of zeros of Jacobi polynomials from different sequences, Electron. Trans. Numer. Anal. 38(2011)317-326.

[4] K.Driver, K. JordaAn, Stieltjes interlacing of zeros of Laguerre polynomials from different sequences, Indag. Math. (NS)21(2011)204-211.

[5] K. Driver, K. JordaAn, Bounds for extreme zeros of some classical orthogonal polynomials, J. Approx. Theory 164 (2012) 1200-1204.

[6] G. SzEgő, Orthogonal Polynomials, fourth ed., vol. XXIII, American Mathematical Society Colloquium Publications, Providence, RI, 1975. 